\title{
Rainbow schlieren measurements in underexpanded jets from square supersonic micro nozzles
}

\author{
Hiroaki Maeda ${ }^{1, *}$, Hikaru Fukuda ${ }^{1}$,Shinichiro $\mathrm{Nakao}^{1}$, Yoshiaki Miyazato ${ }^{1}$, and Yojiro Ishino ${ }^{2}$ \\ ${ }^{1}$ The University of Kitakyushu, 1-1, Hibikino, Wakamatsu-ku, Kitakyushu, 808-0135, Japan \\ ${ }^{2}$ Nagoya Institute of Technology, Gokiso-cho, Showa-ku, Nagoya, 466-8555, Japan
}

\begin{abstract}
The slightly underexpanded free jet issued from a supersonic micro nozzle with a design Mach number of 1.5 and a square shape of $1 \mathrm{~mm} \times 1 \mathrm{~mm}$ at the nozzle exit has been experimentally investigated using the rainbow schlieren deflectometry combined with the computed tomography where the nozzle operating pressure ratio is held constant at 4.0. Density fields in the free jet obtained from the rainbow schlieren deflectometry are the vortex sheet theory proposed by Tam. It is shown that there is good quantitative agreement between experiment and theory. It is shown that the rainbow schlieren deflectometry is a useful tool for studying the three-dimensional structure of shock containing free jets from micro-scale supersonic nozzles.
\end{abstract}

\section{Introduction}

In recent years, the design and fabrication of a microelectro mechanical system (MEMS) has increased the need for an understanding of fluid flow in microscale devices including inkjet printers, small satellite thrusters, critical nozzles at low Reynolds numbers and flowmeters of next-generation fuel cell vehicles (FCV). Especially, the systems will need miniaturization in the space engineering. A detailed and deep comprehension of the flow characteristics through such a device requires the information of vector and scalar quantities in the flow field.

The Reynolds-averaged Navier-Stokes simulation or large eddy simulation often becomes effective tools to investigate the physical properties in various areas such as aerospace and mechanical engineering because of cost curtailment and time saving. However, such numerical simulations include turbulent models to close the governing equations. The capability of these models in simulating a complex physical phenomenon must be ascertained before the numerical results can be accepted. Numerical results are usually validated by comparing them with reliable experimental data. Qualitative flow visualization including schlieren and shadowgraph techniques ${ }^{1}$ is routinely used to evaluate the computed flowfield. Nevertheless, in these techniques, quantitative assessment of physical properties such as the temperature, density, pressure, and velocity in a simulated flowfield are not feasible.

Many researchers often compare simulated schlieren or shadowgraph images directly with those from experiments having the same flow conditions in order to validate the simulation. However, even though a computed schlieren picture is in good agreement with an experimental one, genuine scalar or vector fields cannot always be captured correctly by the computation because conventional schlieren images can only provide integrated information about the density gradient. Therefore, a comparison between experiment and simulation is only meaningful if the numerical results are spatially averaged along the view direction.

In the present study, the three-dimensional density field of the slightly underexpanded supersonic jet issued from a square micro Laval nozzle with a design Mach number of 1.5 and a square shape of $1 \mathrm{~mm} \times 1 \mathrm{~mm}$ at the nozzle exit plane is for the first time clarified by the rainbow schlieren deflectometry ${ }^{2 \sim 4}$ combined with the computed tomography. Also, the three-dimensional free jet flow fields under the same conditions as the experiment are solved using the vortex sheet theory ${ }^{5}$. A quantitative comparison between experiment and theory is carried out to investigate three-dimensional spatial variations of the shock-containing free jet. It is found that the rainbow schlieren deflectometry combined with the computed tomography is a very efficient tool to measure three-dimensional density fields of shock-containing free jets from micro nozzles and to validate numerical simulation codes.

\section{Vortex Sheet Model}

In general, the vortex sheet model ${ }^{5}$ proposed by Tam can quantitatively predict the gross features of a moderately underexpanded jet from an axisymmetric or rectangular nozzle, including the shock-cell spacing, screech frequencies except for higher levels of underexpanded or overexpanded conditions with the formation of a Mach disk or Mach shock inside the jet. A general solution of the vortex sheet shock cell boundary value problem can be found by the method of eigenfunction expansion.

\footnotetext{
*Hiroaki Maeda: x6mba020@eng.kitakyu-u.ac.jp
} 
Figure 1 shows a linear shock cell model for the underexpanded free jet from a square supersonic nozzle with a width $h_{\mathrm{e}}$ at the exit and a design Mach number $M_{\mathrm{d}}$. In the case of the underexpanded jet, the Prandtl-Meyer expansion waves occur from the nozzle lip because the nozzle exit pressure $p_{\mathrm{e}}$ is larger than the back pressure $p_{\mathrm{b}}$. The expansion waves are reflected as the compression waves on the opposite jet boundary and one shock cell is formed.

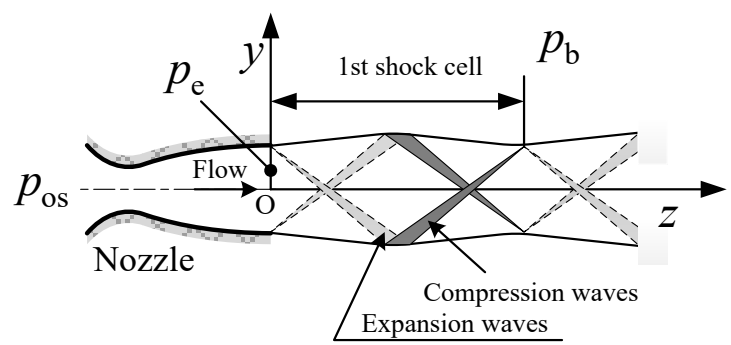

Fig. 1. Vortex sheet model

It is assumed that inside the jet the flow is inviscid and the mixing layer is thin vortex sheet, and outside the jet there is no disturbance. By combining the equation of continuity, the linearized equation of motion and the isentropic equation, it is straightforward to obtain the analytical solution for the pressure $p$ and density $\rho$ inside the jet as follows:

$$
\begin{aligned}
& p(r, z)=\sum_{m=1}^{\infty} \sum_{n=1}^{\infty} A_{m n} \phi_{m n} \cos \left(\lambda_{m n} z\right)+p_{\mathrm{b}} \\
& \rho(r, z)=\rho_{\mathrm{j}}+\frac{p(r, z)-p_{\mathrm{b}}}{a_{\mathrm{j}}^{2}}
\end{aligned}
$$

with

$$
\begin{aligned}
& A_{n m}=\frac{4 \Delta p}{n m \pi^{2}}\left[1-\cos \left(\frac{m \pi h_{e}}{h_{j}}\right)\right]\left[1-\cos \left(\frac{n \pi h_{e}}{h_{j}}\right)\right] \\
& \phi_{m n}=\sin \left[\frac{m \pi}{h_{j}}\left(x+\frac{h_{j}}{2}\right)\right] \sin \left[\frac{n \pi}{h_{j}}\left(y+\frac{h_{j}}{2}\right)\right] \\
& \lambda_{m n}=\frac{\pi}{\left(M_{j}^{2}-1\right)^{\frac{1}{2}}}\left(\frac{m^{2}+n^{2}}{h_{j}^{2}}\right)^{\frac{1}{2}}(m, n=1,2, \\
& \Delta p=p_{e}-p_{b}
\end{aligned}
$$$$
M_{\mathrm{j}}=\sqrt{\frac{2}{\gamma-1}\left\{\left(\frac{p_{\mathrm{os}}}{p_{\mathrm{b}}}\right)^{\frac{\gamma-1}{\gamma}}-1\right\}}
$$$$
\frac{h_{e}}{h_{\mathrm{j}}}=\left(\frac{M_{\mathrm{j}}}{M_{\mathrm{d}}}\right)^{\frac{1}{2}}\left[\frac{2+(\gamma-1) M_{\mathrm{d}}^{2}}{2+(\gamma-1) M_{\mathrm{j}}^{2}}\right]^{\frac{\gamma+1}{4(\gamma-1)}}
$$

where $x$ is the horizontal axis, $y$ the perpendicular axis, and $z$ the longitudinal axis, $\gamma$ the specific heat ratio, $\Delta p$ the pressure difference between $p_{\mathrm{e}}$ and $p_{\mathrm{b}}, n$ and $m$ are the natural numbers, $\phi_{n m}$ the eigenfunctions, and $\lambda_{n m}$ the eigenvalues. Also, $h_{\mathrm{j}}, \rho_{\mathrm{j}}, M_{\mathrm{j}}$, and $a_{\mathrm{j}}$ are the width, density, Mach number, and the speed of sound of the fully expanded jet, which are calculated based on the assumption of the isentropic flow from the nozzle inlet to the back pressure in the downstream of the nozzle exit. This shock-cell model can apply over a range of fully expanded Mach number $M_{\mathrm{j}}$ given by

$$
\left|M_{\mathrm{j}}^{2}-M_{\mathrm{d}}^{2}\right|<1
$$

for underexpanded and overexpanded jet conditions. In addition, Suda and Kaji ${ }^{6}$ investigated unsteady shock behavior in a rectangular underexpanded supersonic jet using a laser schlieren system and found that although the first and second shock cells slightly oscillate across the time-mean location, the third shock cell flaps dynamically with a travelling shock wave in it. Therefore, the shock cell theory appears to be reasonable over the region from the nozzle exit to the end of the 2 nd shock cell.

\section{Rainbow Schlieren Tomography}

The problem of obtaining reliable quantitative information about the flow structure of three-dimensional supersonic jets arises in many industrial fields. Although direct imaging such as the conventional schlieren cannot provide quantitative information about the structure of three dimensional jets, the schlieren $\mathrm{CT}$ which combines the rainbow schlieren deflectometry with so-called computed tomography, often abbreviated as CT must be utilized. Computed tomography was originally developed for use in medical diagnostics with X-rays, but was later adapted for other applications in many scientific fields including physics, chemistry, astronomy, and geophysics, using in many cases other kinds of radiation and even elementary particles. A brief outline of computed tomography for density fields in the three-dimensional jet flows is given by Faris et al. ${ }^{7}$ and Agrawal et al. ${ }^{8}$.

Figure 2 shows a light ray traveling in a cross-section ( $x, y$ plane) of an asymmetric jet issued from a nozzle where the $x, y, z$ rectangular Cartesian coordinate system is used and the $z$ axis is perpendicular to the $x, y$ plane which includes the vector along the optical axis direction of the schlieren system. Also, the $n$ and $n_{a}$ in Fig. 2 indicate the refractive index in the jet cross-section and that of the surrounding air, respectively.

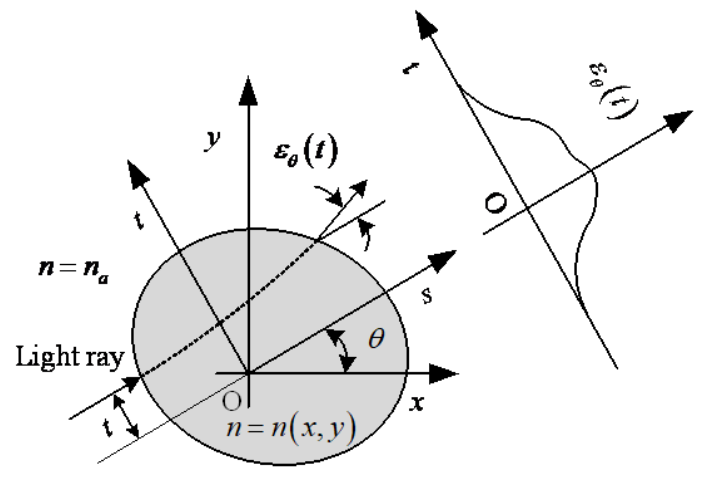

Fig. 2. Projection for tomographic reconstruction 
The refractive index or density fields will now be investigated for a cross section of $z=$ constant. As shown in Fig. 2, let us consider the rotated coordinates $(s, t)$ inclined at an angle $\theta$ away from the fixed-original coordinates $(x, y)$. Then, a light ray traveling in the $s$ direction with an offset of $t$ from the axis $s$ is bent by the interaction with the jet flow and has an angle of inclination $\varepsilon_{\theta}(t)$ with respect to its original path. As the incoming ray is initially parallel to the $s$ axis, the deflection angle $\varepsilon_{\theta}(t)$ after passing through the refractive index field is given by the line integral

$$
\varepsilon_{\theta}(t)=\int_{-\infty}^{\infty} \frac{\partial \eta(s, t)}{\partial t} d s
$$

along the $s$ direction of the partial derivative $\partial \eta / \partial t$ with respect to the $t$ variable of the normalized refractive index difference

$$
\eta(s, t) \equiv \frac{n-n_{a}}{n_{a}}
$$

for small ray deflections. The deflection angle $\varepsilon_{\theta}(t)$ is taken for a range of various angles from $\theta=0$ deg to 180 deg. The task of tomographic reconstruction in the present investigation is to find $\eta(x, y)$ based upon a given knowledge of $\varepsilon_{\theta}(t)$ and then density fields can be obtained through a well-known linear relation between refractive index and density, as described later.

After the one-dimensional Fourier transform of Eq. (10) with respect to the $t$ variable and using the Leibniz rule for differentiation under the integral sign, it reduces to a relation,

$$
\begin{aligned}
& \int_{-\infty}^{\infty} \varepsilon_{\theta}(t) \exp (-i 2 \pi t \zeta) d t= \\
& 2 \pi i \zeta \int_{-\infty}^{\infty} \int_{-\infty}^{\infty} \eta(s, t) \exp (-i 2 \pi t \zeta) d s d t
\end{aligned}
$$

The transformation from the $(s, t)$-coordinate to $(x, y)$ coordinate for the integral of the right-hand side of Eq. (12) yields the following form with $u=-\zeta \sin \theta$ and $v=$ $\zeta \cos \theta$

$$
\begin{aligned}
& \int_{-\infty}^{\infty} \int_{-\infty}^{\infty} \eta(s, t) \exp (-i 2 \pi t \zeta) d s d t= \\
& \int_{-\infty}^{\infty} \int_{-\infty}^{\infty} \eta(x, y) \exp [-i 2 \pi(u x+v y)] d x d y
\end{aligned}
$$

This equation expresses the two-dimensional Fourier transform of $\eta(x, y)$. Hence, the inverse Fourier transform of Eq. (12) using the result of Eq. (13) with the convolution theorem leads to

$$
\begin{aligned}
& \qquad \eta(x, y)=\int_{0}^{\pi}\left[\varepsilon_{\theta}(t) * k(t)\right]_{t=-x \sin \theta+y \cos \theta} d \theta \\
& \text { where } k(t) \text { is given by }{ }^{8} \\
& \quad k(t)=\frac{\sin ^{2}\left(\pi t f_{\text {max }}\right)}{\pi^{2} t}
\end{aligned}
$$

with the Nyquist frequency $f_{\max }$ and the symbol * denotes convolution between $\varepsilon_{\theta}(t)$ and $k(t)$.

When deflection data are sampled at a spacing of $\Delta t$, only frequencies below the Nyquist frequency

$$
f_{\max }=\frac{1}{2 \Delta t}
$$

are adequately sampled.

The ray deflection angle $\varepsilon_{\theta}(t)$ is correlated with the focal length $f_{d}$ of a decollimating lens and the ray transverse displacement $d_{\theta}(t)$ at the cut-off plane of the schlieren system and given by

$$
\varepsilon_{\theta}(t)=\frac{d_{\theta}(t)}{f_{d}}
$$

For air there is a simple linear relation between the refractive index $n$ and the gas density $\rho$ :

$$
\rho=\frac{n-1}{K}
$$

where $K$ is the Gradstone-Dale constant. As the $K$ hardly depends on the wavelength of light, the same constant is used for all wavelengths.

Combination of Eqs. (11), (17) and (18) yields threedimensional density fields of shock-containing jets. The more detailed descriptions for the reconstruction process to obtain three-dimensional density fields are reported in a paper of Agrawal et al. ${ }^{8}$.

\section{Experimental Apparatus}

Experiments have been performed in a blow-down supersonic wind tunnel with the jet issued in the quiescent laboratory air. A schematic diagram of the experimental apparatus with a schlieren optical system is shown in Fig. 3. The air supplied by a compressor that pressurizes the ambient air up to $1 \mathrm{MPa}$ is filtered, dried and stored in a high-pressure reservoir consisting of two tanks with a total capacity of $2 \mathrm{~m}^{3}$. The high-pressure dry air from the reservoir is stagnated in a plenum chamber and then discharged into the atmosphere through a test nozzle. The total temperature in the plenum chamber is equal to the room temperature, and the plenum pressure is controlled and maintained constant during the testing by a valve.

A Laval nozzle with a design Mach number of 1.5 was used as the test nozzle and it is schematically shown in Fig. 4. It has a two-dimensional wall contour with a 850 $\mu \mathrm{m}$ height at the throat and $1000 \mu \mathrm{m}$ height at the exit.

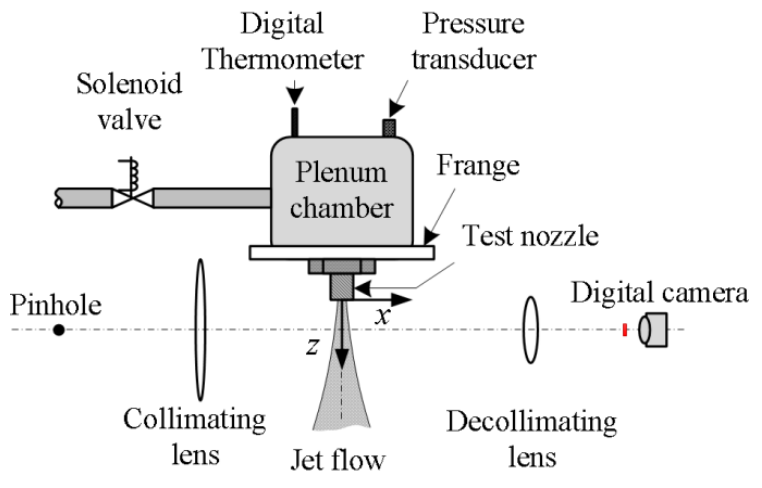

(a) $x-z$ plane

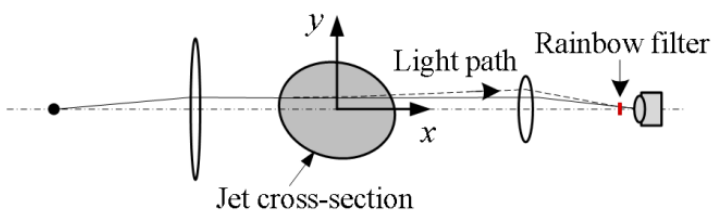

(b) $x-y$ plane

Fig. 3. Schematic diagram of experimental apparatus 


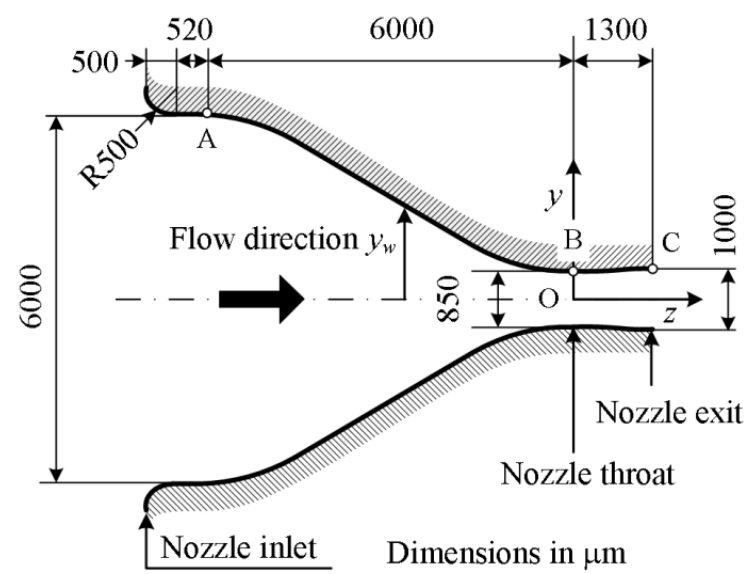

Part A to B: $y_{w}=1288 \sin \left[\frac{\pi}{6000}(z-3000)\right]+1713$

Part B to C: Method of characteristics

Fig. 4. Schematic drawing of test nozzle

The nozzle wall contour over a range of parts A to B is designed to be a sinusoidal curve and that over a range of parts $\mathrm{B}$ to $\mathrm{C}$ is designed by the method of characteristic ${ }^{9}$ to provide uniform and parallel flow in the nozzle exit plane at the design condition.

The jet issued from the nozzle is visualized by the rainbow schlieren deflectometry with a field of view of 30 $\mathrm{mm}$ diameter for a nozzle pressure ratio of $p_{\mathrm{os}} / p_{\mathrm{b}}=4.0$ where $p_{\mathrm{os}}$ is the plenum pressure and $p_{\mathrm{b}}$ is the ambient pressure or back pressure. Also, the ambient pressure, temperature, and density for the present experiment are $p_{\mathrm{b}}$ $=100.5 \mathrm{kPa}, T_{\mathrm{b}}=298.7 \mathrm{~K}$, and $\rho_{\mathrm{b}}=1.17 \mathrm{~kg} / \mathrm{m}^{3}$, respectively. Since the theoretical nozzle pressure ratio for the present nozzle is 3.67 , the nozzle is operated at slightly underexpanded condition.

The rainbow schlieren system consists of railmounted optical components including a $50 \mu \mathrm{m}$ diameter pinhole, a collimating achromatic lens with $100 \mathrm{~mm}$ diameter of $f_{\mathrm{c}}=500 \mathrm{~mm}$ focal length, a decollimating achromatic lens with $30 \mathrm{~mm}$ diameter of $f_{\mathrm{d}}=250 \mathrm{~mm}$ focal length, a computer generated $35 \mathrm{~mm}$ wide slide with color gradation in a $1.4 \mathrm{~mm}$ wide strip, and a digital camera (Nikon D7100) with a $30 \mathrm{~mm}$ diameter focusing lens of $600 \mathrm{~mm}$ focal length. The source image magnification factor defined as the ratio of the source image size to the original source size at the pinhole plane for the present rainbow system can be readily estimated from a relationship of the geometrical optics as $f_{\mathrm{d}} / f_{\mathrm{c}}=0.5$. Therefore, the source image with a $25 \mu \mathrm{m}$ diameter is formed at the focal point of the decollimating lens, wherein a rainbow filter is placed. It should be noted that the resolution and sensitivity of the rainbow schlieren system depend primarily on the size of the source image, the focal length of the decollimating lens, and resolution of color gradients on the rainbow filter ${ }^{2}$.

A continuous $250 \mathrm{~W}$ metal halide light source connected to a $50 \mu \mathrm{m}$ diameter fiber optic cable provides the light input at the pinhole through a $16.56 \mathrm{~mm}$ focal length objective lens. The camera output in the RGB format is digitized by a personal computer with 24 bit color frame grabber. The rainbow filter used in the present experiments is shown in Fig. 5. The filter was fabricated in computer software and then printed digitally on a high resolution $23 \mathrm{~mm}$ color film recorder. It has continuous hue variation from hue $=0$ to $360 \mathrm{deg}$ in a $1.4 \mathrm{~mm}$ wide strip and the origin $y=0$ corresponds to Hue $=200 \mathrm{deg}$. The characteristics of the rainbow filter were performed by traversing the filter automatically in intervals of 0.05 $\mathrm{mm}$ in the $y$ direction at the schlieren cut-off plane before starting experiments. The calibration result is shown as open symbols in Fig. 6. The abscissa is Hue and the ordinate is the transverse ray displacement $d$ from the $x$ axis at the cut-off plane. The solid line indicates a leastsquares regression line of the experimental data using fifth degree polynomials.

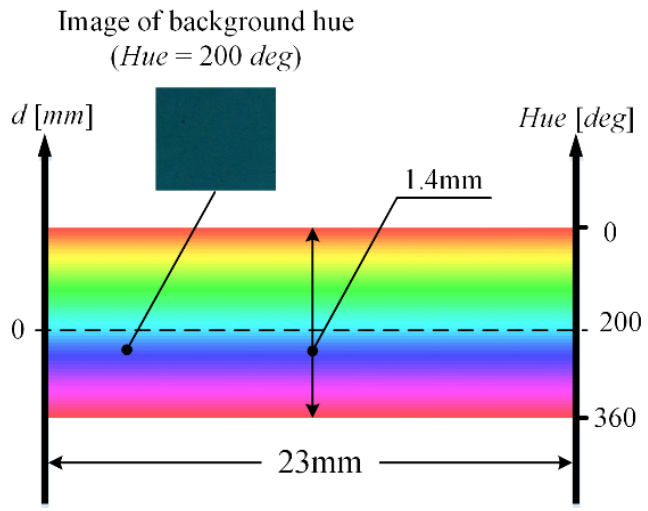

Fig. 5 Rainbow filter image

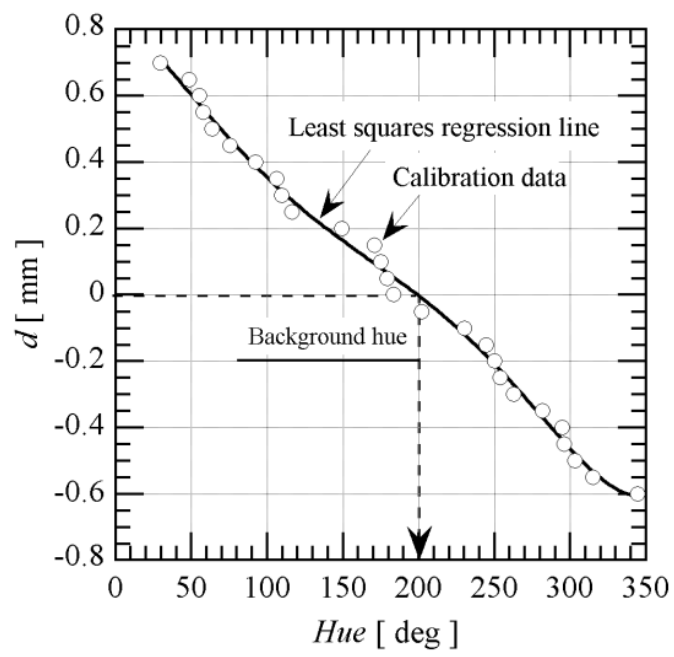

Fig. 6. Calibration curve of rainbow filter

As shown in Fig. 7, the test nozzle is installed inside a pulley with a gear ratio of 1:4 and it can be rotated about the center axis of the nozzle by a stepping motor with a gear ratio of $1: 36$ connected through a timing belt. In the present experiment, rainbow schlieren pictures are acquired over a range of nozzle angular angles from $\theta=0$ deg to -180 deg by rotating the nozzle about its longitudinal axis ( $z$ axis) in equal angular intervals of -5 
deg. These 37 schlieren pictures are used for reconstruction of the jet density field by the schlieren CT method. The jet density profiles obtained from the schlieren $\mathrm{CT}$ method are compared with the vortex sheet model.

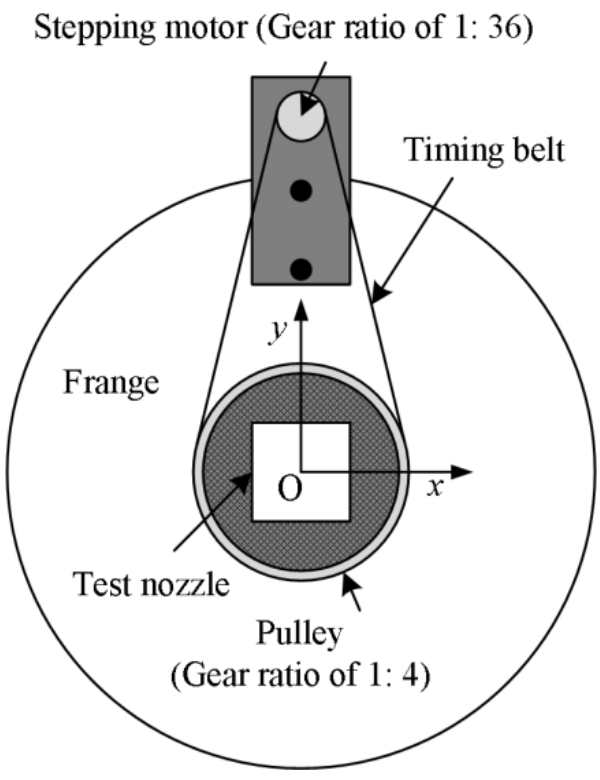

Fig. 7. Nozzle rotating device

\section{Results and Discussion}

A typical rainbow schlieren picture of slightly underexpanded supersonic jet issued from a square micro Laval nozzle is shown in Fig. 8 with the flow from left to right. The ratio $p_{\mathrm{os}} / p_{\mathrm{b}}$ of the plenum pressure $p_{\mathrm{os}}$ to back pressure $p_{\mathrm{b}}$ is held constant at 4.0. As shown on the upper right corner in the picture of Fig. 8, the rainbow filter is placed horizontal with respect to the $z$ axis at the schlieren cut-off plane and the background hue corresponds to the color of the location indicated as the dashed line on the filter image.

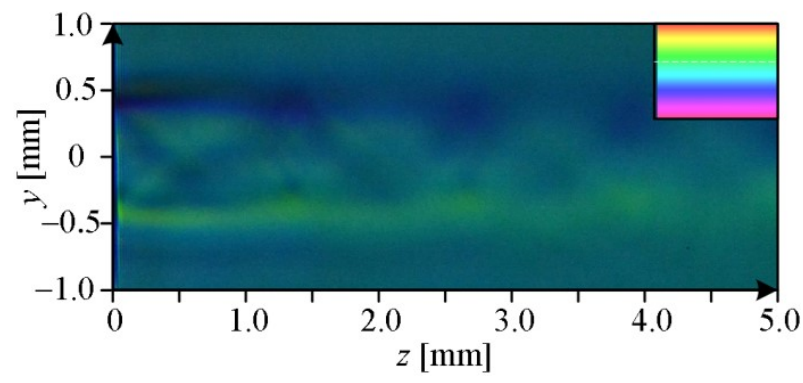

Fig. 8. Rainbow schlieren picture of underexpanded supersonic jet from micro Laval nozzle for $p_{\mathrm{os}} / p_{\mathrm{b}}=4.0$

The schlieren picture has a high resolution of 426 pixels $/ \mathrm{mm}$ and it corresponds to a spatial resolution of around $2.35 \mu \mathrm{m}$ for the present schlieren set up. The schlieren picture of Fig. 8 shows the archetypal structure of underexpanded jet, which is the quasi-periodic shock cell structure, but it is blurred over the full range of the shock cell structure and the jet free boundaries are ambiguous. Therefore, with rainbow schlieren pictures only, it is very difficult to detect significant flow features of micro supersonic jets.

The three-dimensional density field corresponding to the schlieren picture of Fig. 8 can be inferred using the comvolution-backprojection (CBP) algorithm, which is valid even for reconstruction of an asymmetric jet. A density contour plot of the underexpanded micro jet , which includes the jet centerline ( $z$ axis ), is given in Fig. 9 with a color bar representation showing density values and the solid lines with a numeral in the contour indicate isopycnic where experimental data near the nozzle exit plane are excluded in advance for the reconstruction because they include unrealistic density values.
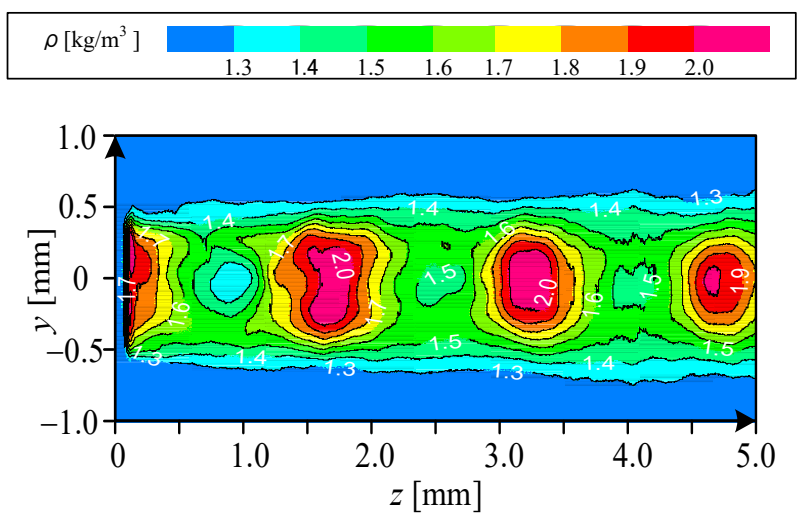

Fig. 9. Density contour plot of underexpanded supersonic jet at $y-z$ plane

Unlike the schlieren picture of Fig. 8, the density contour plot illustrates the various flow features of the shock cell structure quantitatively such as the shape and size of expansion and compression regions, shock cell intervals, jet boundaries, and mixing layers. The density contour of Fig. 9 exhibits nearly symmetry about the jet centerline.

A comparison between experiment and theory for the jet density profile in the streamwise direction at a vertical location of $y=0 \mathrm{~mm}$ is given in Fig. 10 where the blue line shows the density reconstructed from the rainbow schlieren and the red line is calculated from the theory with only the fundamental Fourier mode of $n=m=1$ for Fig. 10(a) and the theory for Fig. 10(b) consists of the Fourier modes added sequentially from $n=m=1$ to $n=$ $m=3$. Also, the left pointing arrow in Fig. 10 shows the density at the nozzle exit plane which is calculated based on the assumption of the isentropic flow from the nozzle inlet to the exit. The density distribution of the theory shows a typical sinusoidal wave form consisting of a smooth repetitive oscillation with a constant wavelength and amplitude. The streamwise locations of the local maxima and minima and the wavelength for the experiment are in good agreement with those for the theory, but there is some difference quantitatively for the density amplitudes. As can be seen in Fig. 10(b), there is a very favorable overall agreement between experiment and theory over the full length of the region measured except for some deviation at the second shock cell where the theoretical density profile is shifted downstream 


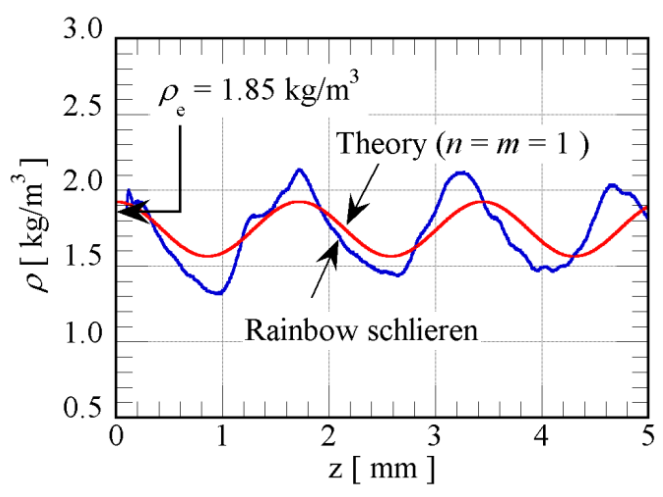

(a) Comparison between experiment and theory with fundamental Fourier mode alone

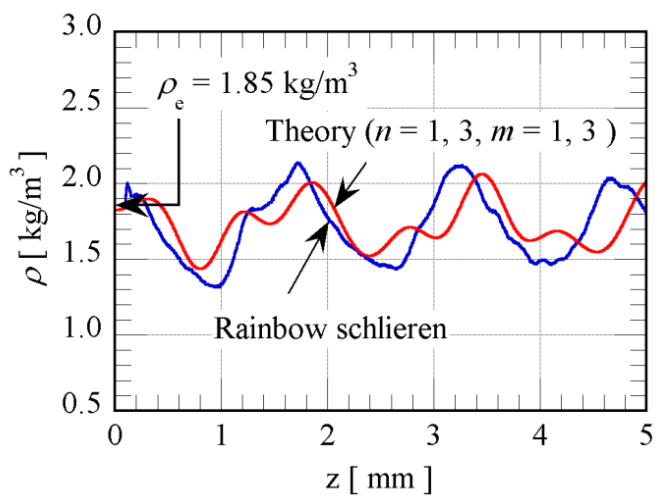

(b) Comparison between experiment and theory with Fourier modes added sequentially from $n=m=1$ to $n=m$ $=3$

Fig. 10. Streamwise density distributions at $y=0$

\section{CONCLUDING REMARKS}

Three-dimensional density fields of the shock containing free jet issued from a square Laval nozzle with a design Mach number of 1.5 were quantitatively obtained by the rainbow schlieren deflectometry combined with the computed tomography based on the convolution backprojection algorithm. As a result, it was found that a density contour plot at any cross-section of the jet can be efficiently inferred from the schlieren CT. Density fields reconstructed from the schlieren $\mathrm{CT}$ are in good quantitative agreement with those from vortex sheet model.

It was demonstrated by continuous color gradation that the density field including the jet axis shows the representative shock-cell structure of a repeated compression and expansion waves. Also, the density profile along the jet centerline exhibits a significant structure consisting of fine-scale spatial oscillations with the wavelengths of the higher-order modes in addition to the fundamental mode.

\section{NOMENCLATURE}

$a_{\mathrm{j}}$ : fully expanded speed of sound, $\mathrm{m} / \mathrm{s}$ $f_{\mathrm{c}}$ :focal length of collimating lens, $\mathrm{mm}$ $f_{\mathrm{d}}$ :focal length of decollimating lens, $\mathrm{mm}$ $h_{\mathrm{e}}$ : width at nozzle exit plane, $\mathrm{mm}$

$h_{\mathrm{j}}$ :fully expanded jet width, $\mathrm{mm}$

Hue : color on rainbow filter, deg

$M_{d}$ : design Mach number, -

$M_{\mathrm{j}}$ : fully expanded jet Mach number, -

$n_{\mathrm{a}}$ :refractive index at surrounding air, -

$p_{\mathrm{e}}$ :static pressure at nozzle exit, $\mathrm{Pa}$

$p_{\text {os }}:$ plenum pressure, $\mathrm{Pa}$

$p_{\mathrm{b}}$ : back pressure, $\mathrm{Pa}$

$T_{\text {os }}$ : plenum temperature, $\mathrm{K}$

$T_{\mathrm{b}}$ :ambient temperature, $\mathrm{K}$

$x, y, z:$ Cartesian coordinate system

$\gamma:$ specific heat ratio, -

$\theta:$ angle at polar coordinate, deg

$\rho_{\mathrm{b}}$ :ambient density, $\mathrm{kg} / \mathrm{m}^{3}$

$\rho_{\mathrm{j}}$ :fully expanded jet density, $\mathrm{kg} / \mathrm{m}^{3}$

\section{REFERENCES}

1. Settles, G. S., Schlieren and Shadowgraph Techniques, Springer, (2001).

2. Al-Ammar, K., Agrawal, A.K., Gollahalli,S.R., and Griffin, D., Application of Rainbow Schlieren Deflectometry for Concentration Measurements in an Axisymmetric Helium Jet, Applied Optics, 25-2, (1998), pp. 89-95.

3. Kolhe,P.S. and Agrawal, A.K., Density Measurements in a Supersonic Microjet Using Miniture Rainbow Schlieren Deflectometry, AIAA J., 47-4, (2009), pp. 830-838.

4. Takano, H., Kamikihara, D., Ono, D., Nakao, S., Yamamoto, H., and Miyazato, Y., Three-Dimensional Rainbow Schlieren Measurements in Underexpanded Sonic Jets from Axisymmetric Convergent Nozzles, $J$. Thermal Science, 25-1, (2016), pp. 78-83.

5. Tam, C.K.W., The Shock-Cell Structures and Screech Tone Frequencies of Rectangular and NonAxisymmetric Supersonic Jets, J. Sound and Vibration, 121-1, (1988), pp. 135-147.

6. Kaji, S., and Nishijima, N., Pressure Field Around a Rectangular Supersonic Jet in Screech, AIAA J., 3410, (1996), pp. 1990-1996.

7. Faris, G.W., and Byer, R. L., Three-Dimensional BeamDeflection Optical Tomography of a Supersonic Jet, Applied Optics, 27-24, (1988), pp. 5202-5212.

8. Agrawal, A.K., Butuk, N.K., Gollahalli, S.R., and Griffin, D., Three-Dimensional Rainbow Schlieren Tomography of a Temperature Field in Gas Flows, Applied Optics, 37- 3, (1998), pp. 479-485.

9. Foelsch, K., The Analytical Design of an Axially Symmetric Laval Nozzle for a Parallel and Uniform Jet, J. Aeronautical Sciences, 16-3, (1949), pp. 161166, and p. 188. 